\title{
Quantifying a Cellular Automata Simulation of Electric Vehicles
}

\author{
Graeme Hill *
}

\begin{abstract}
Within this work a Nagel-Schreckenberg (NS) cellular automata is used to simulate a basic cyclic road network. Results from SwitchEV, a real world Electric Vehicle trial which has collected more than two years of detailed electric vehicle data, is used to quantify the results of the NS automata, demonstrating similar power consumption behavior to that observed in the experimental results. In particular the efficiency of the electric vehicles reduces as the vehicle density increases, due in part to the reduced efficiency of EVs at low speeds, but also due to the energy consumption inherent in changing speeds. Further work shows the results from introducing a spatially restricted speed restriction. In general it can be seen that induced congestion from a spatially transient event propagates back through the road network and alters the energy and efficiency profile of the simulated vehicles, both before and after the speed restriction. Vehicles upstream from the restriction show a reduced energy usage and an increased efficiency, vehicles downstream show an initial large increase in energy usage as they accelerate away from the speed restriction.
\end{abstract}

\section{[1] INTRODUCTION}

For the UK to achieve a sizable reduction in carbon emissions by 2050, it will be necessary for large scale changes to be made. These may be in the form of societal upheavals (mass movement of people to more carbon friendly cities [1]), changes in consumption levels [2] or a technological change. Of the three possibilities shown here, it is a technological change which may be adopted with the minimum of difference to the average person's lifestyle and as such will be the easiest for society to accept.

One such technological change would be the introduction and mass adoption of Electric Vehicles (EV) whether as a hybrid vehicle, purely battery powered vehicle or plug in hybrid. [3]

Given ideal power generation, large scale adoption of electric vehicles will lead to a typical improvement in carbon emissions of approximately $50 \%$ compared to that of an IC vehicle. This is in addition the health benefits from the reduction of pollutants released by vehicles within urban environments.

However, the large scale introduction of EVs, and the effect this would have on transport metrics such as traffic levels and commuter behavior is not an experimentally known quantity, due to the relative scarcity of electric vehicles on the road. Trials exist $([4,5])$ which have examined how EVs are being used within the current environment, but each of these projects is limited in that they can only assess the use of EVs within the current environment and fleet composition, they do not assess the

\footnotetext{
e * G. Hill is with the Transport Operations Research Group, Newcastle University, Newcastle upon Tyne, UK (phone: (44) 0192 222-6428; e-mail: g.a.hill@ncl.ac.uk)
}

use of EVs in vehicle population where they are a significant proportion of the total fleet.

To properly assess the impact of a new technology within society it is necessary to use not only real world studies, but also to use the results of such studies to properly inform simulations. Simulations, when properly conducted, allow for a much wider range of scenarios to be tested. One possible technique for simulation is through the use of Cellular Automata. [6]

A cellular automata is a discrete model where each cell may exist in a number of different discrete states. The transition from one state to another is governed by that model's specific rule set and can either be deterministic, as is the case for Conway's famous Life rule set [7], or stochastic. In addition to the distinction between deterministic and stochastic rule sets the cellular automata may also be reversible [8] but this is generally only possible for the simpler deterministic rule sets.

In recent years cellular automata have begun to find multiple uses in physical simulations including, but not limited to, such topics as cryptography [9], neural simulation [10], and population dynamics. [11]

However, the field of transport simulation is a natural fit for simulation by cellular automata. Cellular automata work at the base level by having single discrete entities which are a representation, within the model, for something within the real world. In a subject like fluid dynamics the cell may correspond to a particular section of space. Within transport studies the cell will typically correspond to either a specific piece of road or a single vehicle. If the vehicle's behavior can be governed by a set of rules then it should be possible to model the behavior of that vehicle using cellular automata.

A lot of previous work has been undertaken using the Nagel-Schreckenberg (NS) [12] model,one of the earliest cellular automata transport models. From a remarkably simple rule set it can reproduce some of the more complicated modes of behavior exhibited by traffic. Although the NS model is not the most sophisticated model, it exhibits enough of the features shown by traffic in the real world to serve as a base starting point for expanding cellular automata to utilize real world EV transport data.

In addition to simply simulating the transport system, the energy usage within the system has also been simulated. [16] For this simulation the theoretical energy usage was compared to the density of the vehicles on the network. The work presented in this project is concentrating on the full energy usage of the electric vehicles using real world data, as modeled by cellular automata, compared to previous work which focused on purely theoretical results and data.

Although it is possible to simulate vehicle with cellular automata there exist other possibilities. Current micro simulation packages, would allow for electric vehicles to be simulated directly in something approaching a real word 
scenario. However, full micro simulation packages are typically computationally intensive, and may not be necessary in all cases. By contrast cellular automata simulations are typically less intensive to run as they do not attempt to model the fine grain behavior of the vehicle, instead they rely on the statistical nature of their processes to accurately recreate the macroscopic behavior in the traffic system. In addition cellular automata are typically easy to run in massive parallel systems and as such can benefit greatly from distributed computing techniques.

\section{[2] The Data And The Model}

\section{A. The Vehicle Data}

The data to realize the quantization of the cellular automata is drawn from the empirical data collected as part of the SwitchEV project. [13]

SwitchEV is a large scale trial in the North East of England and is one of the largest real world data collection projects for electric vehicles. The aims of the project are to accurately assess how electric vehicles are used in a day to day driving cycle by monitoring each vehicle on a second by second basis and assessing the effect of numerous parameters on the EV usage. In addition other data sets are collected to enable a much more complete picture to be built up around the use of EVs. The additional data includes, but is not limited to, road gradients, local weather, traffic congestion parameters through both local SCOOT systems and the open data released by the UK Government on interurban link data [14] and data from charging points (private and public). As well as the hard data sets, multiple questionnaires and focus groups have been undertaken with the EV drivers to determine their thoughts and preconception before the trial, and how they may have altered after their experience of the electric vehicle. [15]

The vehicle monitoring is conducted through the use of multiple data loggers fitted to each vehicle. Typically each logger will monitor the battery usage of the vehicle, the speed and position and also any features of the vehicle, such as lights or air-conditioning, which may contribute to the vehicle's ancillary load.

Specifically from the vehicle's Controller Area Network (CAN) data it is possible to determine the second by second power usage of the electric vehicle. This is simply derived through the instantaneous voltage and current on the battery. It is then possible to construct a heat-map for the vehicle's typical power usage for any particular state of the vehicle. Within this work the power usage of the vehicle was calculated using the speed and acceleration of the vehicle. Other possible factors such as air conditioning, current road gradient, passenger number or ambient temperature were not included due to the lack of representation within current cellular automata traffic models.

\section{B. The Model}

The rule set used in this model is the fundamental Nagel-
Schreckenberg model, a probabilistic irreversible cellular automata model designed to simulate freeway traffic and reproduce some of the phenomenon observed within. Although this model does not exhibit the full range of behaviors which would be exhibited within the real world traffic set, it does reproduce several macroscopic aspects of the traffic which are of interest when studied in conjunction with the experimental EV data.

To initiate the model the vehicle positions are distributed evenly across the road network according to the assigned vehicle density. The vehicles are all initially at rest. Within the NS model each vehicle is updated every time step and simultaneously. For each time step the following rules are applied to each vehicle.

1. (Acceleration) Each car not at its maximum velocity increases its velocity by one unit.

2. (Collision Avoidance) If the distance to the car in front is smaller than the current velocity, then the current velocity is reduced to this distance minus one unit.

3. (Randomization) The speed of the car is reduced by one unit, with a probability of $\mathrm{P}$.

4. (Movement) All cars are advanced by their current speed.

The four conceptual rules above can be condensed down further into 3 main steps:

$$
\begin{array}{ll}
\text { 1. } & v_{i}(t+1) \rightarrow \min \left(v_{i}(t)+1, v_{\text {max }}, d_{i}(t)\right) \\
\text { 2. } & v_{i}(t+1) \rightarrow \max \left(v_{i}(t)-1,0\right) \text { with } P(p)_{\square} \\
\text { 3. } & x_{i}(t+1) \rightarrow x_{i}(t)+v_{i}(t+1)
\end{array}
$$

Where $d_{i}(t)$ is the distance to the next nearest vehicle, $v_{\max }$ is the maximum speed and $P(p)$ is the probability of deceleration.

In general there are multiple different parameters which can be varied to produce different traffic scenarios. For example the maximum speed or the randomization probability may be varied. Although the effect of the maximum speed is obvious the effect of the randomization is less so. Previous work has shown that a random deceleration is needed to induce non deterministic behavior into the model. In addition to the parameters governing the behavior of each individual vehicle within the rule set it is also possible to vary other factors, such as the initial density of cars in the simulation and also the topographical nature of the road. For example, in the simplest form of the simulation, the road is a cyclic loop with vehicles exiting at one end and re-entering at the opposite end, with the same vehicle state. Other possible topographies include sections of road with variable maximum speeds, merging lanes, 
junctions, variable vehicle injection parameters etc.

For this work this simplest form of road, a cyclic homogeneous road was used. Although this is not the most realistic road pattern it does allow the most room for the propagation of patterns through the system and also reduces the need to worry about "end effects" induced by the start and end of the road.

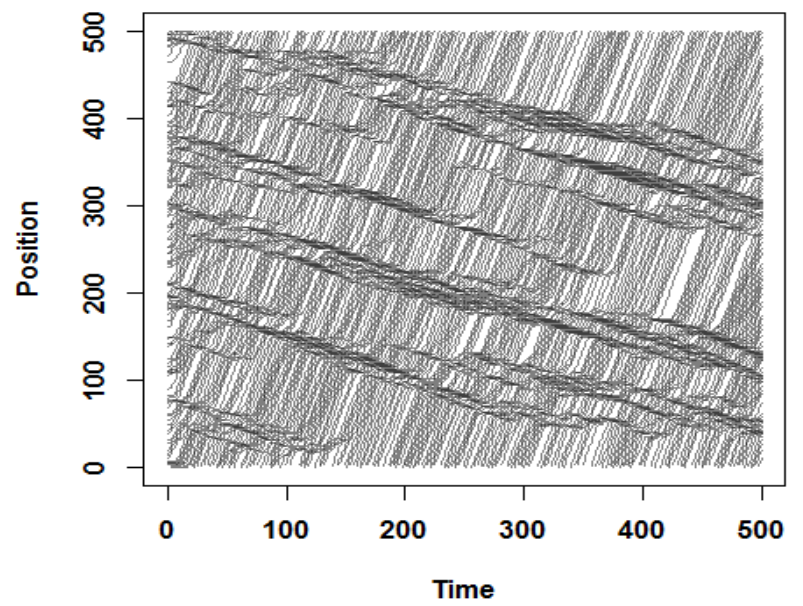

Fig. 1. The spontaneous appearance and subsequent propagation of congestion shock waves forwards in time and backwards through the road network is shown here

In figure 1 it can be seen that the model exhibits the traffic shock flow patterns expected to be observed in a freeway traffic model. The propagation of the shock down the freeway and ahead in time is represented here by the denser patterns which appear with no discernible cause and propagate down and to the right. Although the existence of such shock patterns is no guarantee that the model will accurately simulate other aspects of traffic flow, it is a good indication that there is, at some level, a relationship between the model and real life.

\section{Quantizing the Model}

To assign power consumption to a single vehicle in the simulation it is necessary to categorize the vehicle to allow easy comparison with the real world data. In the NS model each cell is assumed to be $5 \mathrm{~m}$ long with a unit time of approximately 1 second. Hence an in-simulation speed of 1 cell/unit time would translate into a real world speed of 5 $\mathrm{m} / \mathrm{s}$. By assigning real world speeds to the speeds exhibited by the vehicles in the simulation, it is possible to directly assign real world speeds, and hence power consumptions.

In addition to the speed of the vehicle, the power consumption of the electric vehicle is strongly driven by its current acceleration/deceleration profile. EVs in particular have an interesting power consumption for deceleration as they may regain energy when decelerating from a given speed. However, categorizing the power consumption by acceleration and relating this to the simulated electric vehicle is not as straightforward as for speed.
In particular the problem with the NS model is that the acceleration and deceleration are much harsher than would be observed in real life. The typical acceleration/deceleration profile for the EVs ranges from $+/-3 \mathrm{~m} / \mathrm{s}^{2}$ with the majority falling within $+/-1 \mathrm{~m} / \mathrm{s}^{2}$. Within the NS model the vehicles are allowed to accelerate or decelerate by one cell per unit time step. This is equivalent to a speed change of approximately $5 \mathrm{~m} / \mathrm{s}^{2}$, a markedly higher value than that exhibited by the vehicles on the road. Future work may include reducing the size of each time step to allow for more realistic acceleration and deceleration values.

However, currently an assumption is made that the vehicles will only be categorized as accelerating, decelerating or at a steady speed. It is then possible to look at this as a three state system where the driver has chosen one of three possibilities:

\section{Brake Applied \\ 2 Accelerator Applied \\ 3 No Action}

The categorization for the acceleration of the real world data has been achieved by splitting the population for each of the populations previously categorized by speed, into three evenly sized populations, ordered by acceleration magnitude. So the deceleration category will contain the bottom third of the acceleration, the steady speed category will contain the middle third and the acceleration category will contain the top third. It may be possible with future data sets to directly split the data by the user's action

Hence each individual power consumption point will be categorized by two variables, speed and acceleration. From these categories it is possible to gain the current power consumptions for any simulated vehicle in any of the vehicle states by assigning a power consumption state based on that vehicles particular speed and an acceleration state based on the vehicles current acceleration condition.

Statistics for the entire simulation, including such metrics as total power consumption, total efficiency or average, may then be collected and analyzed.

\section{[3] RESULTS}

Previous modeling results suggests that a vehicle density of 0.2 vehicles per spatial unit and a spontaneous deceleration probability of 0.2 are good parameters to allow the simulation to exhibit congestion like behavior.

In general the parameters for the simulation size (both in terms of physical size of the road, and the temporal length for each individual run) were chosen so that the statistical uncertainty of the general behavior of the model (rather than variation within the population) was reduced to an acceptable level. Typically this would occur with a model of length 1000 cells and a 5000 second time frame. 


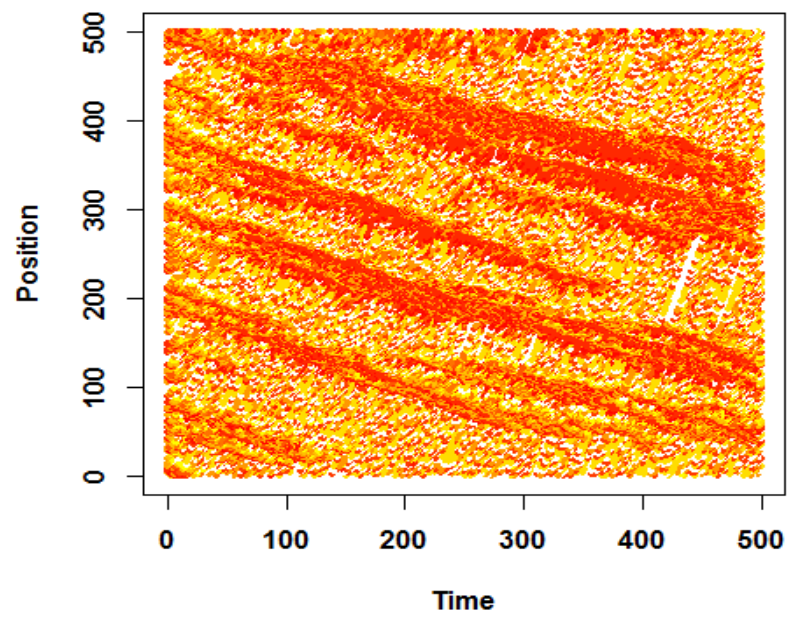

Fig. 2 The power consumption per second for each vehicle at each time point is shown here. The data is drawn from the same simulation as figure 1. Darker areas show points of low power use.

Figure 2 shows that the back propagating shock waves also exist as a feature in the power map for position and time. It can be seen that there is a strong reduction in the overall power usage for any vehicle in a shockwave. However, typically it is not the power consumption which is most important metric for electric vehicles. A more useful metric is the efficiency of the vehicle, which is normally measured in terms of $\mathrm{kWh} / \mathrm{km}$, a dimensional inversion of the mpg form more commonly used for internal combustion vehicles.

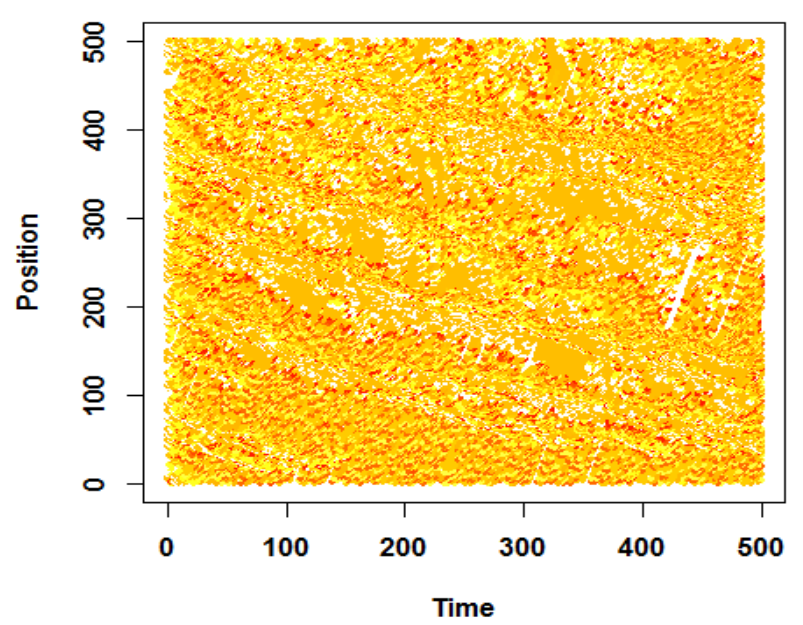

Fig. 3 The vehicle efficiency is shown here. Lighter areas show areas of higher power usage per $\mathrm{km}$ and thus a lower overall efficiency.

It can be seen that the efficiency of the vehicle generally improves at the instigation point of a traffic jam. Initially this may seem a counter intuitive result but in general EVs are less affected by congestion in terms of their efficiency, plus their most efficient speed is in general lower than that of an equivalent IC engined vehicle. So a traffic jam, especially under something closer to freeway conditions, will normally slow the vehicle down and push it into a more efficient speed. It is expected that this would not be the case in urban driving, where the vehicle is operating close to its most efficient speed under free flow conditions, so any congestion will force the vehicle down to a less efficient speed.

To more thoroughly investigate this, the vehicle density was systematically altered and the metrics for the CA analyzed.

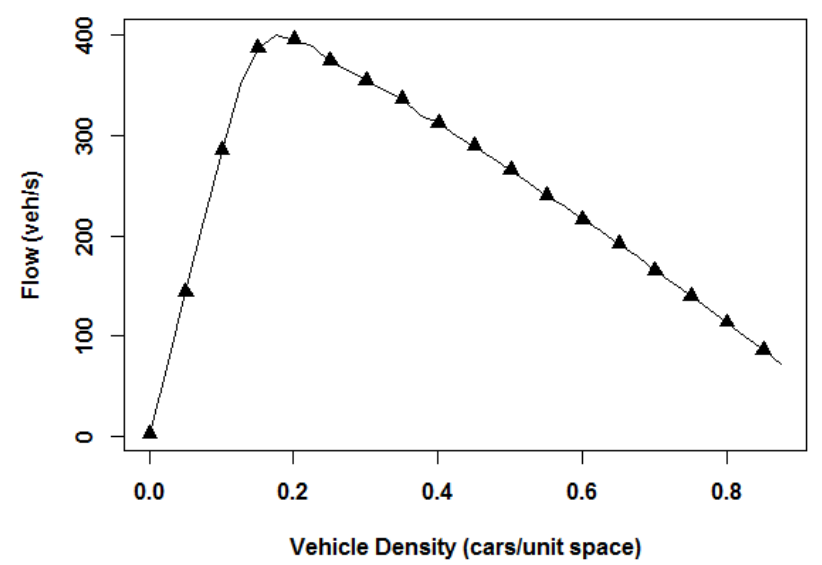

Fig. 4 The fundamental diagram of vehicle flow vs. occupancy is reproduced here.

Figure 4 shows that this simulation will reproduce the fundamental diagram of flow vs. occupancy as observed in both real world data and in other simulation types. Although this model, as shown in other NS models, exhibits an earlier transition into congestion like behavior and a much sharper peak, indicating that the transition phase occurs much quickly than for real world traffic.

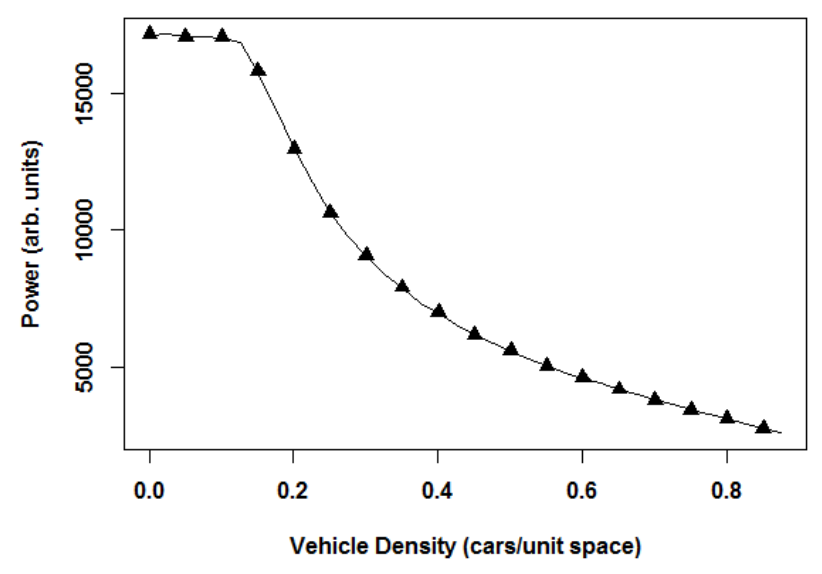

Fig. 5 The instantaneous power usage is shown here.

Figure 5 shows the average instantaneous power usage as a function of vehicle density for a simulation with a 
maximum speed of 4 cell/s. The initial period of steady state power usage corresponds to complete free flow of noninteracting vehicles. Essentially each vehicle is moving at the maximum speed without any forced deceleration from collision avoidance or acceleration back up to maximum speed. As the density of the vehicles increases, the number of interactions between vehicles increases leading to the subsequent decrease in the average speed.

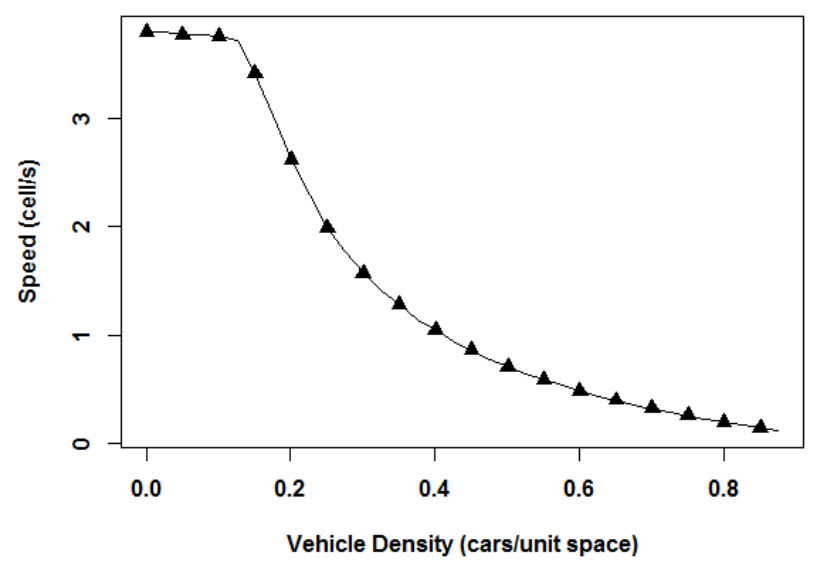

Fig. 6 The average speed for all vehicles can be seen to fall as the vehicle density increases. This matches results in other simulation and in real world scenarios.

Figure 6 shows a similar basic form to that shown in figure 5, due to the strong relationship between instantaneous power and vehicle speed.

The relationship between vehicle efficiency and speed is driven by a combination of two main factors. There is the energy required to produce the vehicle's speed and also the actual speed at which the vehicle is moving. The instantaneous power is the energy expanded to maintain a speed and the speed itself determines the distance over which this energy will be expanded.

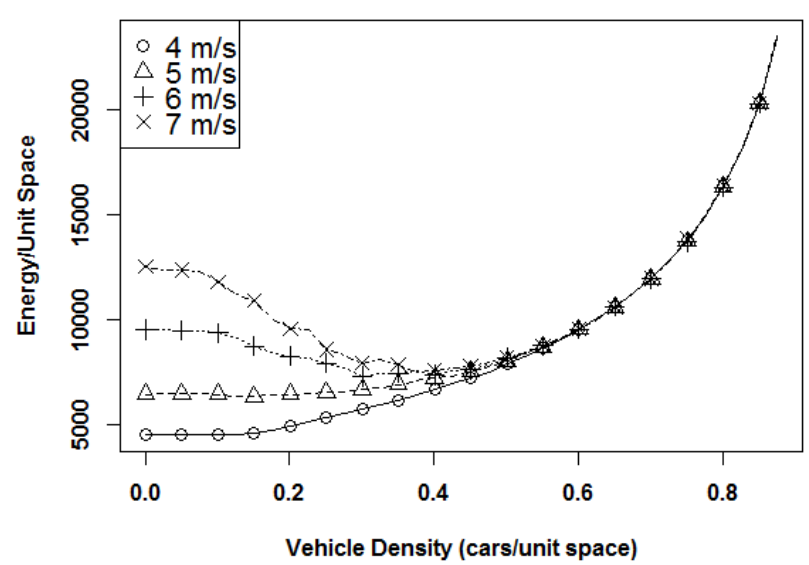

Fig. 7 The vehicle efficiency in terms of $\mathrm{kWh} / \mathrm{km}$ is shown here for four different maximum speeds.

At high speeds a vehicle will require a lot of energy to maintain the high speed, but it will also be covering a lot of ground, whilst at low speeds a vehicle will have a lower power usage but it will cover less distance at this speed. Generally the most efficient speed will lie somewhere between the two extremes. This is behavior common to any system which expends energy to cover distance. At some point the reduction in speed will dominate the efficiency equation, regardless of the reduction in energy usage. Hence at low speeds (which occur at times of high congestion) there will be a greatly reduced efficiency and an increased cost in terms of energy per distance covered.

Figure 7 shows how the efficiency of the vehicles (which here is represented by the metric of average energy used per cell traveled) varies as both the vehicle density and the maximum allowed speed varies. It can be seen that although the four different maximum speeds exhibit the same behavior above the $50 \%$ occupation rate, they exhibit a marked divergence below this point.

This divergence is due to the differing maximum allowable speeds. At low vehicle densities, each vehicle will accelerate up to the different maximum speed allowed by the different model conditions and hence the vehicles will be operating under different power requirements in the four different regimes. However for the different models, if the maximum speed allowed is faster than the most efficient speed (as determined by the real-world data), then any interaction which reduces the average speed of the vehicle will push the vehicle down into a more efficient driving state. Congestion essentially acts as a traffic calming measure to reduce the average speed of the vehicles.

The convergence of the data at points above the 50\% occupation rate is due to each simulation being forced into a lower speed that is purely determined by the congestion of the system rather than the maximum allowed speed of the different models. At this point the different systems exhibit the same behavior as the speed and flow profiles are being dominated by the identical congestion behavior at low speeds.

Although this data is based on an idealized model, parallels do exist within experimental data. In particular studies have shown that the effect of congestion on EVs depends on the type of road where it occurs. For urban congestion the EVs become more inefficient as they are forced into a low-speed/low-efficiency state. For interurban traffic, congestion leads to an improved efficiency as it pushes the EVs from a high-speed/low-efficiency state into a mid-speed/high-efficiency state.

In addition to simulating the features of real world homogeneous traffic it is also possible to simulate less homogeneous environments, such as the influence of a spatially limited speed restriction. 


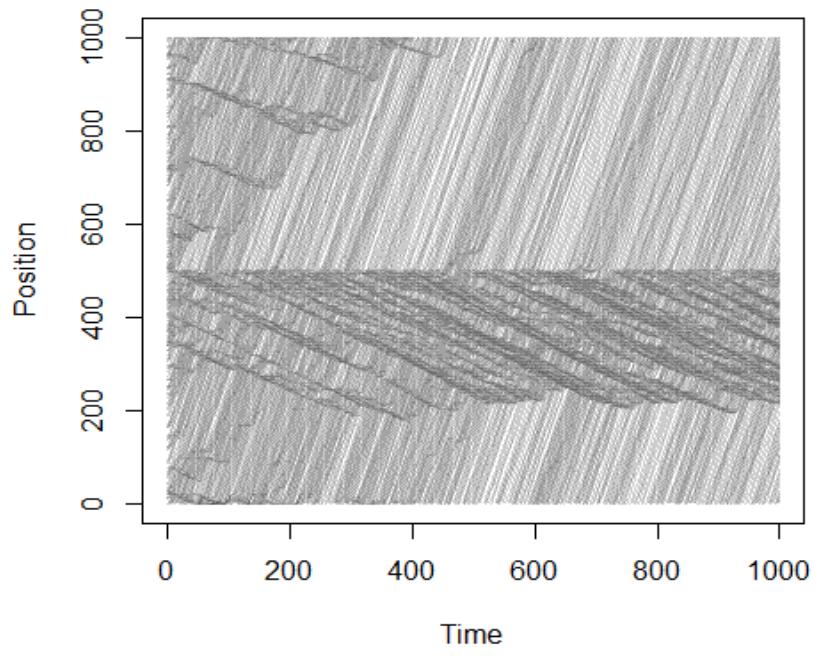

Fig 8 . The position/time figure demonstrates the effect of a single localized speed restriction.

In figure 8 the vehicle position time lines are plotted for a simulation where there is a single point with a restricted speed on the road network. In this example the vehicle speed is restricted to 1 unit/s in the center of the road network.

where

$$
v_{i}(t+1) \rightarrow \min \left(v_{i}(t+1), v_{r e s}\right)
$$

$$
x_{i}(t) \in x_{r e s}
$$

The creation of congestion shock waves can be seen to systematically occur in the area of the speed restriction, see figure 8 , with the congestion propagating upstream from the restriction over a protracted distance. In addition to the systematic creation of new congestion events, the creation of reduced traffic flow downstream from the speed restriction acts as an inhibitor to congestion events downstream by reducing the traffic flow to a level not conducive to congestion events.

To examine the effect of this event on the energy usage and efficiency of electric vehicles specifically, the average power usage and efficiency were collected for each point on the road network. Due to the run-run variation, the data shown here is averaged from multiple runs.
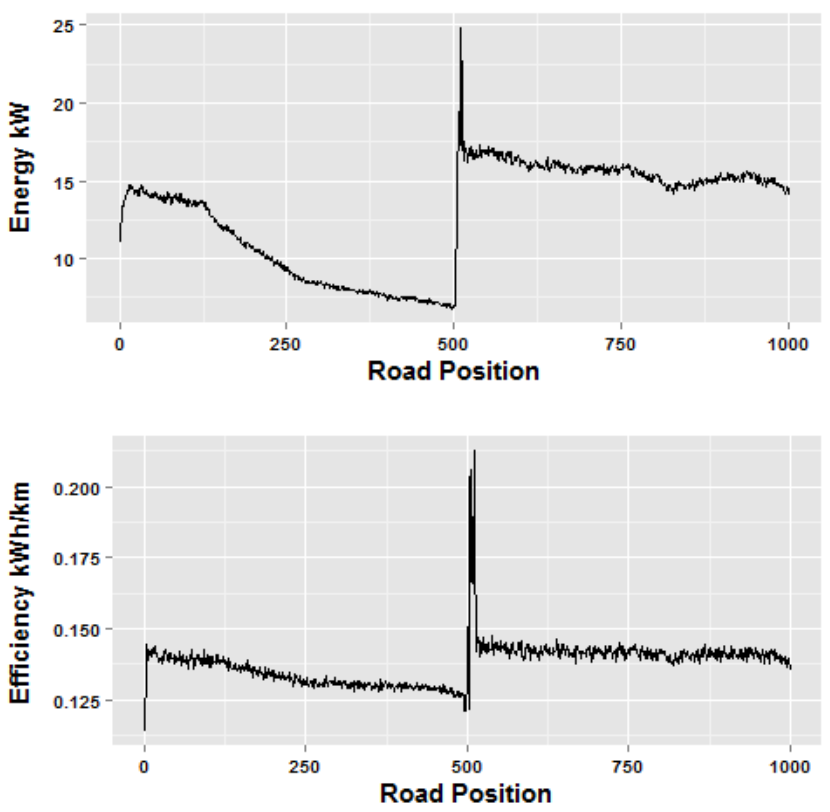

Figure 9 The lines here show the average power usage and efficiency around a cyclic road with a single speed restriction at the half way point.

It can be seen that the effect of the single speed restriction on energy and efficiency is to induce a congestion shock wave which propagates upstream through the traffic environment, altering the average energy usage and efficiency. This data, again, shows results which would be expected from the real world data. In particular there is a reduction in energy usage and a reduction in $\mathrm{kWh} / \mathrm{km}$ as the vehicles slowdown from their less efficient, top speed. There is then a marked increase in power consumption and a reduction in the efficiency of the vehicles after the restriction. The reduction in efficiency and increase in power consumption are both due to the acceleration of the vehicle, once past the speed restriction. However, it can be seen that this is a temporary effect with the vehicles quickly returning to their stead state of power usage and efficiency.

\section{[4] CONCLUSIONS}

Within this work it has been shown that it is possible to quantize a working Nagel-Schreckenberg cellular automaton simulation of freeway traffic with data gained from a real world electric vehicle data set. In particular it is possible to assign the average power consumption for specific speed and acceleration profiles within the experimental data to vehicle states for individual simulated vehicles within the simulation.

Data from the simulations shows similar results to that obtained from the purely experimental results with systematic variations in efficiency and power usage as the vehicle density increases. The variations in efficiency and energy usage match what would be expected from considering the speed and acceleration profiles of the simulated vehicles as analogous to real world vehicles.

In addition to the macroscopic statistics, more detailed examination of heterogeneous road networks demonstrates 
the effect of a single speed restriction; both in the formation of shockwaves upstream from the restriction and also in the change in efficiency and energy usage up and down stream from the restriction. The EVs typically slow down when approaching the congestion event and accelerate away from the congestion event, before settling down to a more steady state driving pattern.

The overall conclusion is that it is possible to quantize cellular automata simulation using real world data, but care must be taken with those points of simulation which do not accurately map onto real world events.

\section{REFERENCES}

[1] R. York, Demographic trends and energy consumption in European Union Nations, 1960-2025, Social Science Research, 36, pp. 855872, 2007

[2] A. Druckman, T. Jackson, The carbon footprint of UK households 1990-2004: A socio-economically disaggregated, quasi-multi-regional input-output model, Ecological Economics 68(7): 2066-2077 2009

[3] T. R. Hawkins, T. R., O. M. Gausen, A. H. Strommen, Environmental impacts of hybrid and electric vehicles-a review, International Journal of Life Cycle Assessment 17(8): 997-1014 2012

[4] CABLED Ultra low carbon car trials in Coventry and Birmingham (http://cabled.org.uk).

[5] Allied Electric, Greener Glasgow's Electric Vehicle Project. (http://www.alliedelectric.co.uk/news/news.cfm/).

[6] S. Wolfram, Statistical Mechanics of Cellular Automata, Reviews of Modern Physics 55(3): 601-644 1983

[7] M. Gardner, The fantastic combinations of John Conway's new solitaire game "life", Scientific American 22,3120-123 1970

[8] J. Kari, Theory of cellular automata: A survey, Theoretical Computer Science 334(1-3): 3-33 2005

[9] M. Tomassini, M. Sipper, M. Perrenoud, On the generation of highquality random numbers by two-dimensional cellular automata, IEEE Transactions on Computers 49 (10): 1146-1151 2000

[10] A. Ilachinski, ” Cellular Automata: A Discrete Universe.” World Scientific. 2001

[11] J. Molofsky, Population Dynamics and Pattern Formation in Theoretical Populations, Ecology 75(1): 30-39. 1994

[12] K. Nagel, M. Schreckenberg, A Cellular Automaton Model for Freeway Traffic, Journal De Physique I 2(12): 2221-2229, 1992

[13] SWITCH-EV (2011 Oct 11) "Switch EV Electric Vehicle Demonstration Project” [Online]. Available: http://vehicletrial.switchev.co.uk/

[14] “Congestion on inter-urban roads" [Online], Available: http://data.gov.uk/dataset/congestion_on_inter-urban_roads

[15] A. Robinson, G. Hill, Y. Hubner, P. T. Blythe "Impact of electric vehicle users' recharging behavior on well to wheel carbon emissions" presented at UTSG 2012, Aberdeeen, U.K.

[16] W. Zhang, W. Zhang, X. Yang, Energy Dissipation in the deterministic and nondeterministic Nagel-Schreckenberg models, Physica A 387: 4657-4664, 2008 